\title{
ESTUDO CINÉTICO DA REAÇÃO DE COMBUSTÃO DE RESÍDUOS METALÚRGICOS COM BIOMASSA*
}

\author{
Manara Nogueira Quintino ${ }^{1}$ \\ José Adilson de Castro² \\ Leonardo Martins da Silva ${ }^{3}$
}

\begin{abstract}
Resumo
No processo de produção de ferro e aço, uma grande quantidade de resíduos é gerada. Esses resíduos e coprodutos contêm consideráveis quantidades de ferro e carbono, que podem ser reaproveitados, ajudando a reduzir a poluição ambiental que pode surgir devido ao descarte inadequado. Este trabalho tem como objetivo avaliar o potencial energético contido em um resíduo siderúrgico, quando misturado à biomassa. Por meio de análise termogravimétrica e cinética (método Coats Redfern) amostras com diferentes proporções de resíduo metalúrgico, biomassa de capim elefante e carvão vegetal foram estudadas. A amostra com menor tamanho de grão e maior quantidade de resíduo foi a que apresentou melhor perda de massa ao final do experimento, apesar de não ter apresentado o melhor resultado antes da faixa isotérmica do experimento. Contudo, a quantidade de capim elefante se mostrou relevante na performance da queima.
\end{abstract}

Palavras-chave: Análise Termogravimétrica; Capim Elefante; Resíduos Metalúrgicos.

\section{KINETIC STUDY OF METALLURGICAL RESIDUES WITH BIOMASS ON COMBUSTION REATION}

\section{Abstract}

During iron and steel making process, a large residue amount is generated. This residue and co products contain considerable quantities of iron and carbon, which can be reused and consequently contributing to environmental pollution reduction,that comes with the inappropriate discard. The aim of this study is to evaluate the energy potential contained in metallurgical residue when mixed with elephant grass biomass and charcoal. Through thermogravimetric analysis and kinect study (Coats - Redfern method), samples contained different proportions of metallurgical residue, elephant grass biomass and charcoal were evaluated. The sample with smaller particle size and bigger residue amount showed the best mass loss in the end of the experiment, although it did not show-the best result before isothermal period. Nerverthless, the elephant grass biomass amount seemed relevant in the performance burning.

Keywords: Thermogravimetric Analysis; Elephant Grass Biomass; Metallurgical Residue

1 Mestranda em Engenharia Mecânica, Universidade Federal Fluminense, Volta Redonda, Rio de Janeiro e Brasil.

2 Professor, Ph.D. Programa de Pós Graduação em Engenharia Metalúrgica, Escola de Engenharia Industrial Metalúrgica de Volta Redonda, Universidade Federal Fluminense, Volta Redonda, Rio de Janeiro, Brasil.

3 DSc. Engenharia Metalúrgica, Programa de Pós Graduação em Engenharia Metalútgica, Escola de Engenharia Industrial Metalúrgica de Volta Redonda,Universidade Federal Fluminense, Volta Redonda, Rio de Janeiro, Brasil. 


\section{INTRODUÇÃO}

O setor metalúrgico é um grande produtor de resíduos, e, pelas normas cada vez mais severas de antipoluição, os responsáveis devem encaminhá-lo para a reutilização ou descarte adequado. A recuperação dos valores metálicos contidos nos resíduos sólidos e co produtos do setor metalúrgico passou a ser muito importante tanto do ponto de vista das restrições legais impostas pelas legislações relativas à proteção do meio ambiente, como do ponto de vista econômico, por contribuírem para a compensação dos custos de instalação e operação de equipamentos antipoluição instalados nas usinas. [1]

A maior parte dos resíduos sólidos ricos em ferro é reciclada via sinterização ou pelotização. Embora a reciclagem da maior parte (80\%) destes resíduos via sinterização seja uma prática siderúrgica comum, esta não é a mais recomendada por diminuir a permeabilidade do leito da carga e consequentemente a perda da produtividade da sinterização [2]. A utilização no processo de pelotização também não é indicada, pois a adição destes resíduos aumenta o consumo de energia [3].

Pesquisas têm sido realizadas ao longo dos anos para a reutilização desse tipo de resíduo sólido juntamente com carvão, assim como misturas de carvão com componentes metálicos, a fim de analisar propriedades catalíticas desses componentes no desempenho da combustão do carvão [3]. Zhang et. al. [4] realizaram um estudo dos efeitos nos valores caloríficos de carvões com alto e baixo poder de coqueificação, quando misturados a aditivos de queima de carvão utilizando a calorimetria de combustão. Os autores concluíram que estes aditivos não possuem efeito significativo nos valores caloríficos de ambos os carvões, porém foi-se visto que são capazes de mudar o curso da oxidação do carbono pela ação da catálise, reduzir a energia de ativação aparente e acelerar a queima/oxidação do grafite. Ma et. al. [5] investigaram o efeito catalítico de $\mathrm{Fe}_{2} \mathrm{O}_{3}, \mathrm{MnO}_{2}$ e $\mathrm{BaCO}_{3}$ quando misturados a cinzas com alto teor de carvão por meio de análise termogravimétrica. Os resultados mostraram que os catalisadores melhoram as características de combustão e reduziram a temperatura de ignição. Zou e Zhao [3] estudaram o comportamento de pós que continham ferro (hematita, pó de alto-forno, pó de conversor de aciaria e lama de aciaria) no comportamento da combustão do carvão por meio de análise termogravimétrica. Foi observado que a hematita, o pó 
de aciaria e a lama de aciaria foram capazes de diminuir a ignição do carvão pulverizado, aumentar a taxa de combustão e o comportamento combustão completa.

A análise térmica pode fornecer informações valiosas sobre 0 comportamento da combustão. Tais ferramentas, como TGA e DSC, também tem se mostrado efetivas para o estudo dos parâmetros característicos da combustão do carvão, sendo largamente aplicados para o estudo da catálise desta reação $[3,5,6$, $7,8,9,10,11]$.

Neste estudo utilizaram-se carvão vegetal, carvão de capim elefante e resíduo metalúrgico e estes foram misturados em diferentes proporções e estas misturas foram analisadas por termogravimetria. Com base nesses dados, os cálculos cinéticos foram realizados para quantificar as taxas de conversões aparentes e compará-las e quantificar os efeitos catalíticos dos óxidos e metais presentes no resíduo.

\section{MATERIAIS E MÉTODOS}

\subsection{Materiais e preparação da amostra}

O resíduo utilizado nesta pesquisa foi doado por uma usina siderúrgica localizada na região sudeste do Brasil, sendo este o pó de coletor de alto forno no estado sólido, gerado no sistema de despoeiramento do alto forno. A análise química foi realizada pela própria empresa de acordo com as normas internas e são apresentadas na Tabela 1. Percebe-se que a maior parte do resíduo é composta por óxidos, sendo o mais abundante o óxido de ferro.

Tabela 1: Análise Química do Pó de Alto Forno

\begin{tabular}{|c|c|c|c|c|c|c|c|c|c|c|c|c|c|c|}
\hline $\begin{array}{l}\text { MATERIAL } \\
(\%) \\
\end{array}$ & $\begin{array}{c}\mathrm{Fe} \\
\text { TOTAL }\end{array}$ & $\mathrm{Fe}_{2} \mathrm{O}_{3}$ & $\mathrm{FeO}$ & $\mathrm{Fe}_{\text {metálico }}$ & $\mathrm{SiO}_{2}$ & $\mathrm{Al}_{2} \mathrm{O}_{3}$ & $\mathrm{MgO}$ & C & $\mathrm{CaO}$ & $\mathrm{Na}_{2} \mathrm{O}$ & $\mathrm{SO}_{3}$ & $\mathrm{~Pb}$ & $\mathrm{P}_{2} \mathrm{O}_{5}$ & $\mathrm{ZnO}$ \\
\hline PÓ DE & & & & & & & & & & & & & & \\
\hline $\begin{array}{c}\text { COLETOR } \\
\text { DE AF\#3 }\end{array}$ & 33,88 & 37,49 & 8,05 & 1,34 & 10,29 & 2,66 & 2,19 & 30,57 & 3,3 & 0,047 & 0,678 & 0,041 & 0,078 & 0,484 \\
\hline
\end{tabular}

As biomassas utilizadas foram o carvão de capim-elefante e carvão vegetal. Foram escolhidos esses materiais por serem finos, conterem alta reatividade, e que em geral precisam ser aglomerados para sua utilização. O carvão vegetal utilizado é composto totalmente por eucalipto, sendo este tipo de madeira o mais utilizado para 
a produção de carvão para os setores siderúrgico e metalúrgico [12]. A análise química do carvão de capim elefante utilizado é apresentada na Tabela 2.

Tabela 2: Composição química do carvão de capim elefante

\begin{tabular}{ccccc}
\hline Constituintes & $\begin{array}{c}\text { Carbono } \\
\text { Fixo }\end{array}$ & Voláteis & Cinzas & Enxofre \\
\hline$\% \mathrm{p} / \mathrm{p}$ & 56 & 32,7 & 11,1 & 0,22 \\
\hline \multicolumn{5}{c}{ Fonte: Adaptado de $[13]$} \\
\hline
\end{tabular}

O resíduo foi peneirado em peneiras vibratórias e a fração passante utilizada foi: $0,044 \mathrm{~mm}$ até $0,088 \mathrm{~mm} ; 0,088 \mathrm{~mm}$ até $0,125 \mathrm{~mm}$ e $0,125 \mathrm{~mm}$ até $0,210 \mathrm{~mm}$. Foram utilizadas porções de $2 \%, 4 \%$ e $6 \%$ de resíduo, juntamente com 12,5\%, 25\% e $37,5 \%$ de capim elefante e carvão vegetal, em amostra de $4 \pm 0,2 \mathrm{mg}$, pesadas em balança de precisão. A composição de cada amostra é apresentada na tabela 3.

Tabela 3: Matriz de planejamento das misturas

\begin{tabular}{cccc}
\hline Amostra & Resíduo (\%) & Capim Elefante (\%) & $\begin{array}{c}\text { Granulometria } \\
(\mathbf{m m})\end{array}$ \\
\hline $\mathbf{2}$ & 6 & 12,5 & $0,044-0,088$ \\
$\mathbf{3}$ & 2 & 37,5 & $0,044-0,088$ \\
$\mathbf{4}$ & 6 & 37,5 & $0,044-0,088$ \\
$\mathbf{5}$ & 2 & 12,5 & $0,125-0,210$ \\
$\mathbf{6}$ & 6 & 12,5 & $0,125-0,210$ \\
$\mathbf{7}$ & 2 & 37,5 & $0,125-0,210$ \\
$\mathbf{8}$ & 6 & 37,5 & $0,125-0,210$ \\
$\mathbf{9}$ & 4 & 25 & $0,088-0,125$ \\
\hline
\end{tabular}

\subsection{Experimentos de Combustão}

Todos os experimentos foram realizados em equipamento de análise termogravimétrica (Q50 da TA Instruments). A taxa de aquecimento utilizada para todas as amostras foi de $15^{\circ} \mathrm{C} / \mathrm{min}$, da temperatura ambiente até atingir $800^{\circ} \mathrm{C}$, permanecendo $25 \mathrm{~min}$ na isoterma. A atmosfera inerte foi ar com fluxo de $50 \mathrm{~mL} / \mathrm{min}$. A massa da amostra foi monitorada constantemente em relação a temperatura. 


\subsection{Análise Cinética}

Neste estudo utilizou-se o método de Coats-Redfern para calcular a energia de ativação $A$ e o fator pré-exponencial $E$ com base nas curvas de TGA [6]. Este método baseia-se no fato das reações de combustão sejam reações de primeira ordem.

Usualmente, para os estudos cinéticos, a taxa de conversão é expressa pela seguinte equação:

$$
\frac{d \alpha}{d t}=k f(\alpha)(1)
$$

Esta equação representa a taxa de conversão, $d \alpha / d t$, a uma temperatura constante em função da perda de massa do reagente e da constante de velocidade. $k$ representa a constante da taxa de reação e $f(\alpha)$ é um modelo de mecanismo de reação. A conversão de a é definida pela equação 2:

$$
\alpha=\frac{m_{0}-m}{m_{0}-m_{f}}(2)
$$

Onde $\mathrm{m}$ representa a massa do reagente e os subscritos 0 e $f$ representam a quantidade no inicio e no fim, respectivamente. A constante $k$ é calculada utilizandose a expressão de Arrhenius:

$$
k(T)=A \exp \left(-\frac{E}{R T}\right)
$$

Combinando as equações 1 e 3 a taxa de reação pode ser escrita da seguinte maneira:

$$
\frac{d \alpha}{d t}=A \exp \left(-\frac{E}{R T}\right) f(\alpha)(4)
$$

Para a combustão do carvão:

$$
f(\alpha)=(1-\alpha)(5)
$$

Para processos não isotérmicos, como os estudados com o auxilio do TGA, substituindo a taxa de aquecimento $\beta$, onde $\beta=d T / d t$, chega-se a seguinte expressão: 
A solução da integral da equação 6 é:

$$
\frac{d \alpha}{d T}=\frac{A}{\beta} \exp \left(-\frac{E}{R T}\right)(1-\alpha)(6)
$$

$$
\ln \left(-\frac{\ln (1-\alpha)}{T^{2}}\right)=\ln \left(\frac{A R}{\beta E}\right)-\frac{E}{R T}(7)
$$

Com base no resultado do gráfico de $\ln \left[-\ln (1-\alpha) / T^{2}\right]$ versus $100 / T$, utiliza-se a regressão linear para calcular os valores de E e A.

\section{RESULTADOS E DISCUSSÃO}

\subsection{Comparação TG da perda de massa até a temperatura máxima do experimento}

A Figura 1 apresenta a curva de perda de massa versus temperatura, sendo definida até o momento de temperatura máxima do experimento, não considerando a parte isotérmica.

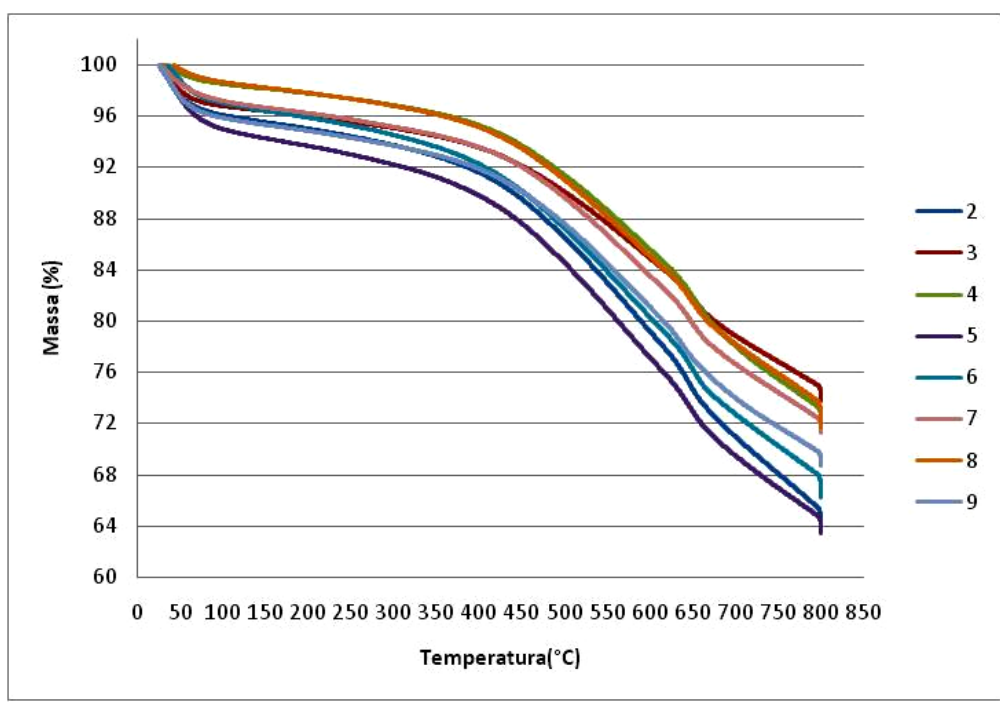

Figura 1: Curvas de TGA até a temperatura máxima

De acordo com a Figura 1, as amostras que obtiveram melhores resultados foram a de número 5, 2 e 6 respectivamente. Apesar das amostras 8 e 4 terem iniciado com o pior resultado (até cerca de $450^{\circ} \mathrm{C}$ ), a amostra 3 foi a que apresentou menor perda de massa ao atingir a temperatura máxima. As amostras 3 e 7 percorreram um caminho semelhante até cerca de $450^{\circ} \mathrm{C}$, o qual a curva 7 apresenta um aumento na velocidade da reação, ocasionando em uma maior perda de massa a partir desta temperatura. As curvas 6 e 9 apresentam perda de massa similar até cerca de $400^{\circ} \mathrm{C}$, onde há uma intersecção com a curva 2, que a partir desse ponto apresenta uma perda de massa maior em relação as outras, 
apresentando redução de massa inferior apenas a da amostra 5, a qual obteve a maior perda de massa até atingir a temperatura máxima.

As amostras 3 e 7 diferem apenas na granulometria do resíduo, isso significa que esta diferença no tamanho do grão se torna significativa a partir de $450^{\circ} \mathrm{C}$, onde a amostra 7 passa a ter uma perda de massa maior. O mesmo não pode ser constatado quando se compara as amostras 8 e 4 , pois ambas as curvas se sobrepõe por quase todo o experimento.

\subsection{Comparação TG da perda de massa na faixa isotérmica}

A figura 2 apresenta as curvas de TGA para todas as amostras, ao final do experimento (25min de isoterma).

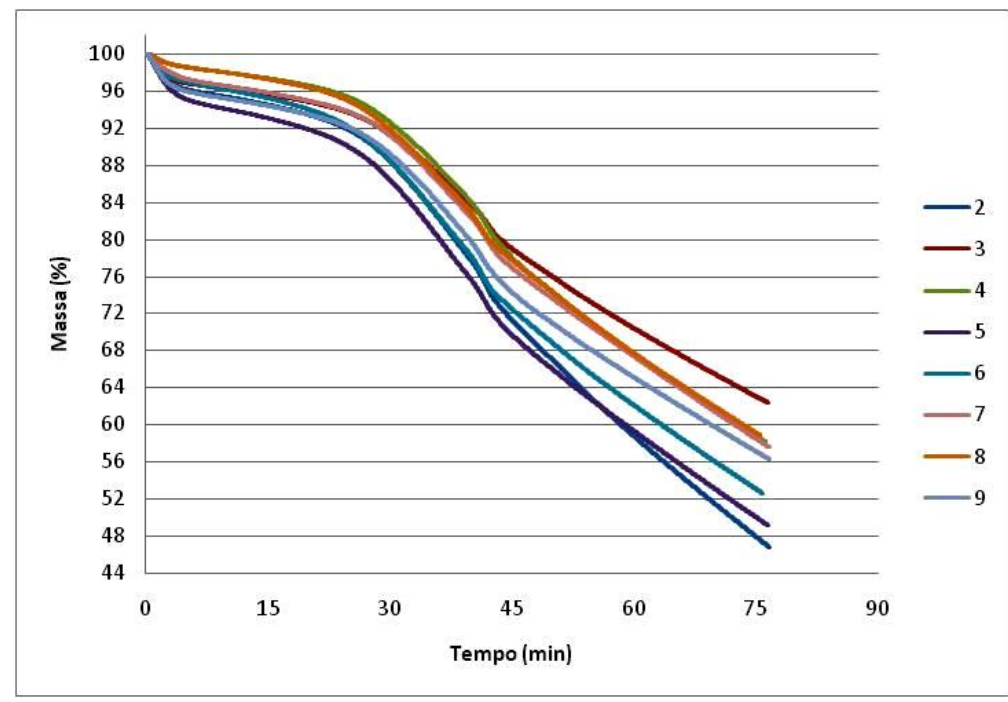

Figura 2: Curvas de TGA no tempo total de experimento

Ao final do experimento, a massa restante de cada amostra foi respectivamente: $46,7 \%, 62,3 \%, 58,1 \%, 49,1 \%, 52,5 \%, 57,2 \%, 58,8 \%$ e $56,3 \%$. De acordo com a Figura 4, as amostras 2, 5 e 6 foram as que obtiveram os melhores resultados, com 46,7\%, $49,1 \%$ e $52,5 \%$ de massa ao final do experimento. A amostra 5 teve um melhor resultado até cerca de 50 min de experimento, ocorrendo então uma intersecção desta curva com a curva da amostra 2 e esta apresentou uma maior perda de massa ao final. As amostras de número 4, 7 e 8 apresentaram resultados muito similares, principalmente após $45 \mathrm{~min}$ de experimento. A amostra que apresentou pior resultado foi a de número 3, não chegando a queimar $40 \%$ de massa.

Com relação perda de massa versus quantidade de capim elefante, como pode ser observado na Figura 2, a ordem da amostra que perdeu maior quantidade 
de massa, para a que perdeu menor quantidade foi $2,5,6,9,7,4,8$ e 3 . As três amostras com menor quantidade de capim elefante $(12,5 \%)$ foram as que apresentaram melhor resultado, seguidas da amostra com quantidade intermediária (25\%) e as que apresentaram pior resultado foram as com maior quantidade dessa biomassa $(37,5)$. Com base nesse fato, conclui-se que quanto maior a quantidade de capim elefante, menor é a capacidade de perda de massa da amostra.

\subsection{Análise Cinética}

De acordo com as curvas de perda de massa para a amostra 2 e 5, que foram apresentadas na figura 4, foi possível dividir os estágios da reação de combustão em diferentes faixas de temperatura, desconsiderando a variação até $50^{\circ} \mathrm{C}$ por se tratar de evaporação de água e calibração do equipamento. As tabelas 4 e 5 apresentam os dados cinéticos para as amostras 2 e 5 para diferentes faixas de temperatura.

Tabela 4: Constantes Cinéticas Amostra 2

\begin{tabular}{|c|c|c|c|}
\hline \multicolumn{4}{|c|}{ Amostra 2} \\
\hline $\begin{array}{c}\text { Faixa de } \\
\text { Temperatura }\end{array}$ & A & $E / R$ & $\mathrm{~K}$ médio \\
\hline $50^{\circ} \mathrm{C}-450^{\circ} \mathrm{C}$ & 0,00702 & 14,640 & 0,00649 \\
\hline $450^{\circ} \mathrm{C}-650^{\circ} \mathrm{C}$ & 0,03290 & 12,590 & 0,03215 \\
\hline $650^{\circ} \mathrm{C}-675^{\circ} \mathrm{C}$ & 0,04720 & 14,260 & 0,04620 \\
\hline $675^{\circ} \mathrm{C}-800^{\circ} \mathrm{C}$ & 0,04262 & 13,520 & 0,04223 \\
\hline
\end{tabular}

Tabela 5: Constantes Cinéticas Amostra 5

Amostra 5

\begin{tabular}{cccc}
\hline $\begin{array}{c}\text { Faixa de } \\
\text { Temperatura }\end{array}$ & A & E/R & $\begin{array}{c}\mathbf{K} \\
\text { médio }\end{array}$ \\
\hline $\mathbf{5 0 ^ { \circ } \mathbf { C } - \mathbf { 1 0 0 } ^ { \circ } \mathbf { C }}$ & 0,01793 & 12,06 & 0,01517 \\
\hline $\mathbf{1 0 0}^{\circ} \mathbf{C}-\mathbf{2 7 5}^{\circ} \mathbf{C}$ & 0,00492 & 13,96 & 0,00455 \\
\hline $\mathbf{2 7 5}^{\circ} \mathbf{C}-\mathbf{4 7 5}^{\circ} \mathbf{C}$ & 0,01254 & 14,23 & 0,01908 \\
\hline $\mathbf{4 7 5}^{\circ} \mathbf{C}-\mathbf{6 2 5}^{\circ} \mathbf{C}$ & 0,03558 & 12,66 & 0,03477 \\
\hline $\mathbf{6 2 5}^{\circ} \mathbf{C}-\mathbf{6 5 0}^{\circ} \mathbf{C}$ & 0,05845 & 11,79 & 0,05738 \\
\hline $\mathbf{6 5 0}^{\circ} \mathbf{C}-\mathbf{6 7 5}^{\circ} \mathbf{C}$ & 0,05316 & 12,37 & 0,05217 \\
\hline $\mathbf{6 7 5}^{\circ} \mathbf{C}-\mathbf{8 0 0}^{\circ} \mathbf{C}$ & 0,04266 & 13,07 & 0,04192 \\
\hline
\end{tabular}

De acordo com as tabelas 4 e 5, o fato de terem sido calculados diferentes valores cinéticos em cada faixa de temperatura significa que não ocorre somente um tipo de reação durante todo o experimento. Esse fato explica o motivo pelo o qual 
ocorrem intersecções entre as curvas de perda de massa em diferentes temperaturas, o que demonstra que, de acordo com a faixa de temperatura uma amostra apresenta maior perda de massa em relação à outra, o que não significa necessariamente que este resultado permanecerá constante até 0 final do experimento.

De acordo com a Tabela 4, a amostra 2 atinge a sua velocidade máxima na faixa de $650^{\circ} \mathrm{C}-675^{\circ} \mathrm{C}$ e essa velocidade média é ligeiramente reduzida quando o experimento atinge $800^{\circ} \mathrm{C}$. Ambas as velocidades são as maiores durante 0 experimento para essa amostra, o que demonstra que a partir de $650^{\circ} \mathrm{C}$ a perda de massa se torna mais efetiva.

Quando se compara as velocidades para as amostras 2 e 5 apresentadas nas tabelas 4 e 5, percebe-se que as velocidades médias encontradas nas diferentes faixas de temperatura para a amostra 5 são maiores que as velocidades encontradas para a amostra 2 , exceto para a faixa de $675^{\circ} \mathrm{C}-800^{\circ} \mathrm{C}$. Nessa faixa a amostra 2 apresenta uma maior velocidade de reação, o que explica a intersecção das curvas de perda de massa, apresentada na figura 2. Dessa forma, os resultados cinéticos condizem com os dados experimentais.

\section{CONCLUSÃO}

Amostras com diferentes proporções de capim elefante, capim vegetal e resíduo de pó de alto forno (em diferentes granulometrias) foram analisadas por meio de análise termogravimétrica. De forma geral, pode - se destacar os seguintes pontos:

- Ao final dos experimentos, a fração de massa restante para as amostras foi respectivamente: $46,7 \%, 62,3 \%, 58,1 \%, 49,1 \%, 52,5 \%, 57,2 \%, 58,8 \%$ e $56,3 \%$. A amostra 2 e 5 foram as que apresentaram melhores resultados, restando menos de $50 \%$ de massa.

- A amostra 3 foi a que apresentou pior perda de massa, não chegando a perder $40 \%$ de massa; a amostra 2, com menor granulometria e maior porcentagem de resíduo, foi a que apresentou melhor perda de massa ao final do experimento.

- Quando comparadas as constantes cinéticas das amostras 2 e 5, percebe-se que depois de $675^{\circ} \mathrm{C}$ a velocidade média da amostra 2 é maior que a 5 , o que justifica a intersecção destas curvas após $50 \mathrm{~min}$ de experimento.

- De forma geral, os valores da amostra 5 para o fator pré-exponencial são menores do que para a amostra 2, o que se justifica a amostra 5 tenha apresentado melhor resultado até a temperatura máxima do experimento ser atingida. 
- Com relação a granulometria, com exceção da amostra 2, as amostras que melhor apresentaram resultados foram as de maior tamanho de grão. Contudo, nota-se que quanto maior a quantidade de capim elefante na amostra, mais lento será o processo de queima.

\section{Agradecimentos}

Os autores agradecem a Coordenação de Aperfeiçoamento de Pessoal de Nível Superior (CAPES) pela bolsa de Mestrado do primeiro autor.

\section{REFERÊNCIAS}

1 Carvalho, Pedro Sérgio Landim de, Pedro Paulo Dias Mesquita,Elizio Damião Gonçalves de Araújo. 2015 "Sustentabilidade da siderurgia brasileira: eficiência energética, emissões e competitividade." BNDES Setorial, Rio de Janeiro

2 Takano, Cyro, Et Al. "A Reciclagem De Resíduos Siderúrgicos Sólidos." Seminário Nacional Sobre Reuso/Reciclagem De Resíduos Sólidos Industriais (2000).

3 Zou, C.,Zhao, J. Investigation Of Iron-Containing Powder On Coal Combustion Behavior. Journal Of The Energy Institute, 2016.

4 Zhang Li. M., Tan Z. C., Wang S. D., And Wu D. Y. Combustion Calorimetric And Thermogravimetric Studies Of Graphite And Coals Doped With A Coal-Burning Additive. Thermochimica Acta, Vol. 299, No. 1-2, Pp. 13-17, 1997.

5 Ma, B. G., Li, X. G., Xu, L., Wang, K., \& Wang, X. G. (2006). Investigation on catalyzed combustion of high ash coal by thermogravimetric analysis. Thermochimica Acta, 445(1), 19-22

6 Li, X. G., Ma, B. G., Xu, L., Luo, Z. T., \& Wang, K. (2007). Catalytic effect of metallic oxides on combustion behavior of high ash coal. Energy \& Fuels, v. 21, n.5, p.26692672.

7 GONG, Xuzhong; GUO, Zhancheng; WANG, Zhi. Reactivity of pulverized coals during combustion catalyzed by $\mathrm{CeO} 2$ and Fe2O3. Combustionand Flame, v. 157, n. 2, p. 351$356,2010$.

8 Yin, K., Zhou, Y. M., Yao, Q. Z., Fang, C., \& Zhang, Z. W. Thermogravimetric analysis of the catalytic effect of metallic compounds on the combustion behaviors of coals. Reaction Kinetics, Mechanisms and Catalysis, 106(2), 369-377, 2012

9 YU, L. Y.; LI, P. S. Thermogravimetric analysis of coal and sludge co-combustion with microwave radiation dehydration. Journal of the Energy Institute, v. 87, n. 3, p. 220-226, 2014. 
10 Cheng, J., Zhou, F., Xuan, X., Liu, J., Zhou, J., \& Cen, K. Cascade chain catalysis of coal combustion by $\mathrm{Na}-\mathrm{Fe}-\mathrm{Ca}$ composite promoters from industrial wastes. Fuel, v.181, p. 820-826, 2016.

11 Cheng, J., Zhou, F., Xuan, X., Liu, J., Zhou, J., \& Cen, K. Comparison of the catalytic effects of eight industrial wastes rich in $\mathrm{Na}, \mathrm{Fe}, \mathrm{Ca}$ and $\mathrm{Al}$ on anthracite coal combustion. Fuel, v. 187, p. 398-402, 2017.

12 Barcellos, Daniel Camara. Caracterização do carvão vegetal através do uso de espectroscopia no infravermelho próximo. Tese de Doutorado - Universidade Federal de Viçosa. 2007, $139 f$.

13 Rocha, Elisa Pinto da. Avaliação Numérica e Experimental da redução em Forno de Cuba utilizando Pelotas Convencionais e Autorredutoras produzidas com Biomassa de Capim Elefante. Tese de Doutorado - Universidade Federal Fluminense. 2016, 207 f. 